\title{
A computational model for fiber-reinforced composites: hyperelastic constitutive formulation including residual stresses
}

\author{
N.-K. Jha ${ }^{1}$, J. Reinoso ${ }^{\mathrm{a}, *}$, H. Dehghani ${ }^{\mathrm{b}}$, J. Merodio $^{\mathrm{b}}$ \\ ${ }^{a}$ Grupo de Elasticidad y Resistencia de Materiales, School of Engineering. Universidad de Sevilla, Camino de los \\ Descubrimientos $s / n, 41092$, Seville, Spain \\ ${ }^{b}$ Department of Continuum Mechanics and Structures, Escuela de Caminos, Canales y Puertos, Universidad Politécnica de \\ Madrid, 28040, Madrid, Spain
}

\begin{abstract}
The computational modelling of fiber-reinforced structures is of prime importance for automotive products like tires, airsprings or hoses and for biomechanical applications like arteries. In the first part of the paper, we outline the development of invariant-based constitutive models suitable for the fiber-reinforced structure within the context of the phenomenological theory. The polyconvex strain energy function of fiber composites is adopted from the literature and split into the energy of the fiber, matrix, and the fiber-matrix interaction respectively. Experimental data from the literature are used to validate the model. In the second part of the paper, the residual stress and damage effects are incorporated into the constitutive relations by exploiting an invariant-based representation and using an isotropic/anisotropic damage formulation, respectively. It is assumed that residual stress arises in fiber-matrix composites due to manufacturing processes and affects the overall lifetime. The proposed formulation is implemented into commercial finite element code ABAQUS/Standard and examined by means of several applications, showing a high robustness and reliability.
\end{abstract}

Keywords: Anisotropic material; Residual Stress; Fiber-Matrix composites; Damage Mechanics

\section{Introduction}

Fiber-reinforced composite materials have been extensively used in many engineering components and system ranging from tires, air-springs or hoses, among many applications. For instance, tire belts are generally composed of several layers with different orientations which provide the desired load bearing capacity for pneumatic tires [1]. Another application where fiber-reinforced materials are widely used is the case of arteries. In particular, for arteries systems, several layers of fiber reinforcement are located at the matrix, which are covered with an matrix layer internally and externally in order to ensure the structural integrity of the component. A cross section of an artery is depicted in Fig. 1 where it can be observed that the constitutive parameters such as the fiber angle, the matrix layer thickness, and other additional factors notable affect the overall life of the artery system.

Consequently, one of the main characteristics of the engineering/mechanical products mentioned above, whereby fiber-reinforced composites are employed, regards their inherent complexity in terms of internal material arrangement and mechanical behavior in service conditions. This aspect is also motivated by their heterogeneity since these composites are generally composed of fiber, matrix and adhesive materials for the final assembly of the component. This multi-material character, with significantly different physical properties among the constituents, makes the mechanical understanding of such systems an arduous task.

\footnotetext{
* Corresponding authors

Email addresses: niraj.kumar.jha@ibnm.uni-hannover.de (N.-K. Jha), jreinoso@us.es (J. Reinoso )
} 
Within the context of the Finite Element Method (FEM), first attempts for modelling fiber-reinforced composites relied on the use of shell models with different material descriptions [2, or the implementation of the so-called Equivalent Single Layer (ESL) [3] approach to account for the resulting average properties. However, material and geometrical nonlinearities of matrix provoked that classical elastic laminate theories became rather inaccurate for capturing the actual response of such composites under general loading conditions.

In view of the previous limitations, different modelling concepts have been posteriorly developed in order to analyze the fiber-reinforced composite materials, which include nonlinearities from geometrical and mechanical signature into their corresponding formulations; cf. [4, ㅎ, 6, 17, where smeared constitutive material formulations based on rebar elements are proposed. The main idea of rebar formulation concerns the use an element as a host element and overlay extra reinforcing elements describing the fiber layers in the host element. The host element represents the matrix material whereas the embedded element describes the reinforcing material. Thus, from the numerical standpoint, the element stiffness matrix of the host-embedded system $\mathbf{K}=\mathbf{K}_{\mathrm{L}}+\mathbf{K}_{\mathrm{NL}}$ for linear and non-linear part is given by respectively,

$$
\mathbf{K}_{\mathrm{L}}=\int_{v} \mathbf{B}_{\mathrm{L}}^{\mathrm{T}} \mathbb{C}_{\mathrm{m}} \mathbf{B}_{\mathrm{L}} \mathrm{d} v-\sum_{1}^{n_{f l}} \int_{v_{f l}} \mathbf{B}_{\mathrm{L}}^{\mathrm{T}} \mathbb{C}_{\mathrm{m}} \mathbf{B}_{\mathrm{L}} \mathrm{d} v_{f l}+\sum_{1}^{n_{f l}} \int_{v_{f l}} \mathbf{B}_{\mathrm{L}}^{\mathrm{T}} \mathbb{C}_{\mathrm{fl}} \mathbf{B}_{\mathrm{L}} \mathrm{d} v_{f l},
$$

and

$$
\mathbf{K}_{\mathrm{NL}}=\int_{v} \mathbf{B}_{\mathrm{NL}}^{\mathrm{T}} \mathbf{S} \mathbf{B}_{\mathrm{NL}} \mathrm{d} v
$$

In Eq. 1. $\mathbb{C}_{\mathrm{m}}$ and $\mathbb{C}_{\mathrm{fl}}$ stand for the constitutive response of matrix and the fiber-layer respectively, $\mathbf{B}_{\mathrm{L}}$ is the deformation matrix (discrete version of the strain-displacement compatibility operator) with respect to the shape functions of the host elements, $n_{f l}$ is the number of embedded fiber layers in the element; $v$ and $v_{f l}$ are the current volumes of the element and fiber layers respectively. In Eq. 2 , $\mathbf{B}_{\mathrm{NL}}$ is the nonlinear strain-displacement transformation matrix where $\mathbf{S}$ corresponds to the second Piola Kirchoff stress.

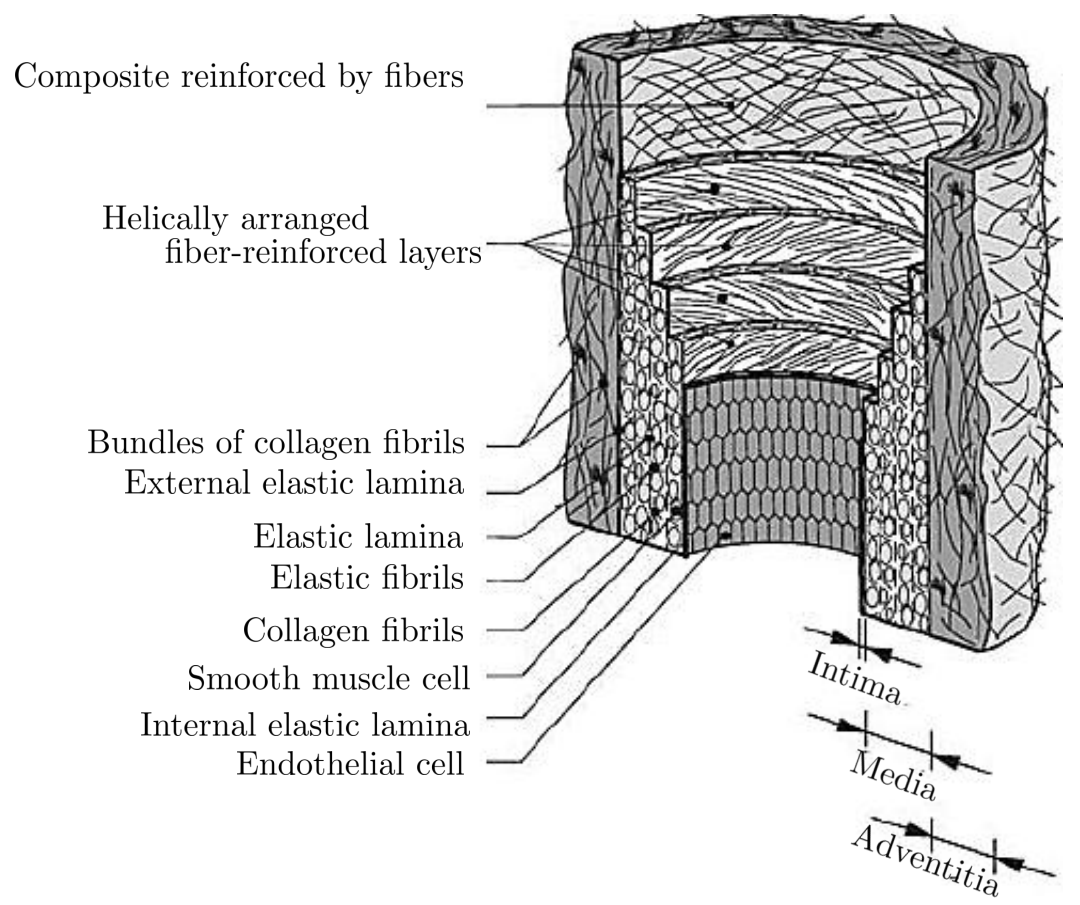

Figure 1: The hierarchical structure of an arteries 
An alternative approach to model embedded fibers into the soft matrix exploits the invariant-based tensorial representation according to the Spencer's theory [8]. Basically, this invariant formulation incorporates the anisotropic character of fiber-reinforced composites by means of the use of a preferred direction via an unit vector field $\mathbf{a}_{\mathbf{0} i}$ ( $i \in 1,2$ for two family of fibres for instance). Therefore, the material anisotropy is defined through the introduction of the structural tensor, $\mathbf{M}_{\mathbf{0} i}=\mathbf{a}_{\mathbf{0} i} \otimes \mathbf{a}_{\mathbf{0}} i$, into the free energy function of fiber-matrix composites as an additional argument. with this method, many variants type of continuum models have been developed in the last years for arteries [9, 10, fiber-matrix composites 1, 11, short fiber reinformed polymers for mechanical and coupled thermo-mechanical analysis [12, and long fiber reinforced composites [13, to quote a few of them. However, the vast majority of the previous models usually neglect the role of the presence of residual stresses.

The incorporation of residual stress fields into novel material models has received a great deal of attention in the last years due to its importance in practice. In particular, some authors have adopted the multiplicative decomposition of the deformation gradient, whereby the inelastic part endows the residual stress field [14, 15]. Alternative approaches have relied on invariant-based representations, as that derived in [16. Though this topic is of high impact in many engineering areas, the consistent treatment of inelastic effects in fiberreinforced matrix composites due to the concomitant presence of residual stresses and damage events has not been extensively covered up to now.

In light of the previous arguments, the primary goal of this research is twofold. The first part of the paper is concerned with the development of several phenomenological constitutive models for fiber-matrix composites that can be applied to products such as tires, air-springs or hoses, among others. The proposed material models are formulated within the thermodynamically consistent framework, which accounts for the material anisotropy using invariant-based tensorial representation [8], and they are validated by means of a benchmark application. The second salient aspect of the present investigation is focused on the incorporation of residual stresses into the constitutive relations that arise due to manufacturing processes in a consistent and efficient manner. To account for such effects, we exploit the seminal formulation proposed by Merodio and coauthors [16, 17, which also admits a tensorial-based treatment within the constitutive formulation in a compact and efficient manner.

The research paper is organized as follows. The phenomenological invariant-based anisotropic constitutive model is detailed in Sect. 2. The validation study on a unit cube is provided in Sect. 3 in order to demonstrate the constitutive response of fiber-matrix composites. The incorporation of the residual stress field on the overall stress and damage capabilities are derived in Sect. 4. Moreover, it can be inferred from the constitutive formulations that the residual stresses are non-uniform and geometry dependent, leading to inhomogeneous elastic response. The constitutive modelling of residually-stressed continuum configuration is presented in Sect. 4. The application of the current model to a cylindrical and cubical system and its extension to anisotropic materials is detailed in Sect. 5. Finally, the main conclusions of this research are drawn in Sect. 6.

\section{Constitutive formulation}

In this Section, we present the basic concepts of nonlinear Continuum Mechanics (Sect 2.1) that are required for the description of finite strain material models, and the different anisotropic constitutive models for the hyperleastic (isotropic and anisotropic) materials herewith considered (Sect 2.2.

\subsection{Fundamentals and basic notation}

This section outlines the fundamental concepts and basic notation of the current study. Following standard arguments, let $\mathcal{B}_{0} \subset \mathbb{R}^{3}$ and $\mathcal{B}_{t} \subset \mathbb{R}^{3}$ to denote the reference and current configurations at time steps $t=0$ and $t$, respectively, see Fig. 2. In both configurations, the corresponding individual material points are denoted by $\mathbf{X} \in \mathcal{B}_{0}$ and $\mathbf{x} \in \mathcal{B}_{t}$. The motion of the body is characterized by the nonlinear mapping operator $\varphi: \mathcal{B}_{0} \times[0, t] \rightarrow \mathbb{R}^{3}$, where $[0, t]$ denotes the time interval elapsed. The displacement vector at material point level is given by: $\mathbf{u}:=\mathbf{x}-\mathbf{X}$. The deformation gradient $\mathbf{F}$ that represents the linear map 
between tangent vectors in the reference and current configurations is defined as

$$
\mathbf{F}:=\frac{\partial \varphi(\mathbf{X}, t)}{\partial \mathbf{X}}=\mathbf{1}+\mathbf{H}(\mathbf{X}, t) ; \mathbf{H}(\mathbf{X}, t)=\nabla_{\mathbf{X}} \mathbf{u}(\mathbf{X}, t)
$$

where 1 stands for the second order identity tensor and $\nabla_{\mathbf{X}}[\bullet]$ identifies the gradient of the magnitude $\bullet$ with respect to the reference setting. The Jacobian of the transformation $J=\operatorname{det}[\mathbf{F}]$ complies with the following restriction $\mathrm{J}>0$. The symmetric right and left Cauchy-Green deformation tensors, $\mathbf{C}$ and $\mathbf{b}$, respectively, and the Green-Lagrange strain tensor, E, are defined as

$$
\mathbf{C}:=\mathbf{F}^{\mathrm{T}} \mathbf{F} ; \quad \mathbf{b}:=\mathbf{F F}^{\mathrm{T}} ; \quad \mathbf{E}:=\frac{1}{2}[\mathbf{C}-\mathbf{1}] .
$$

The local form of the balance of linear momentum, which governs the initial boundary value problem (IBVP) of the deformation process of the body, neglecting the the inertia terms is given by

$$
\operatorname{Div} \mathbf{P}(\mathbf{X}, t)+\overline{\mathbf{\Upsilon}}(\mathbf{X})=\mathbf{0} \quad \text { in } \mathcal{B}_{0}
$$

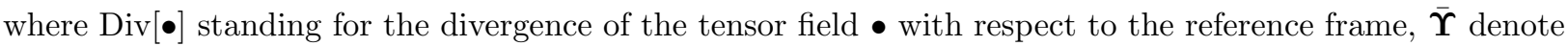
the body forces (per unit reference volume) of the continuum. In Eq. (5) $\mathbf{P}$ stands for the first Piola-Kirchhoff stress tensor, which can be associated with the the symmetric second Piola-Kirchhoff stress tensor, $\mathbf{S}$ as follows: $\mathbf{S}=\mathbf{F P}$. The complete (IBVP) is defined with the consideration of suitable boundary conditions in terms of displacements $\overline{\mathbf{u}}: \Gamma_{u} \times[0, t] \rightarrow \mathbb{R}^{3}$ and nominal tractions $\overline{\mathbf{T}}: \Gamma_{t} \times[0, t] \rightarrow \mathbb{R}^{3}$, which are prescribed on the corresponding subsets of the body boundary. Moreover, the balance of angular momentum leads to the symmetry condition of the second Piola-Kirchhoff stress tensor in the reference configuration $\mathbf{S}=\mathbf{S}^{\mathrm{T}}$.

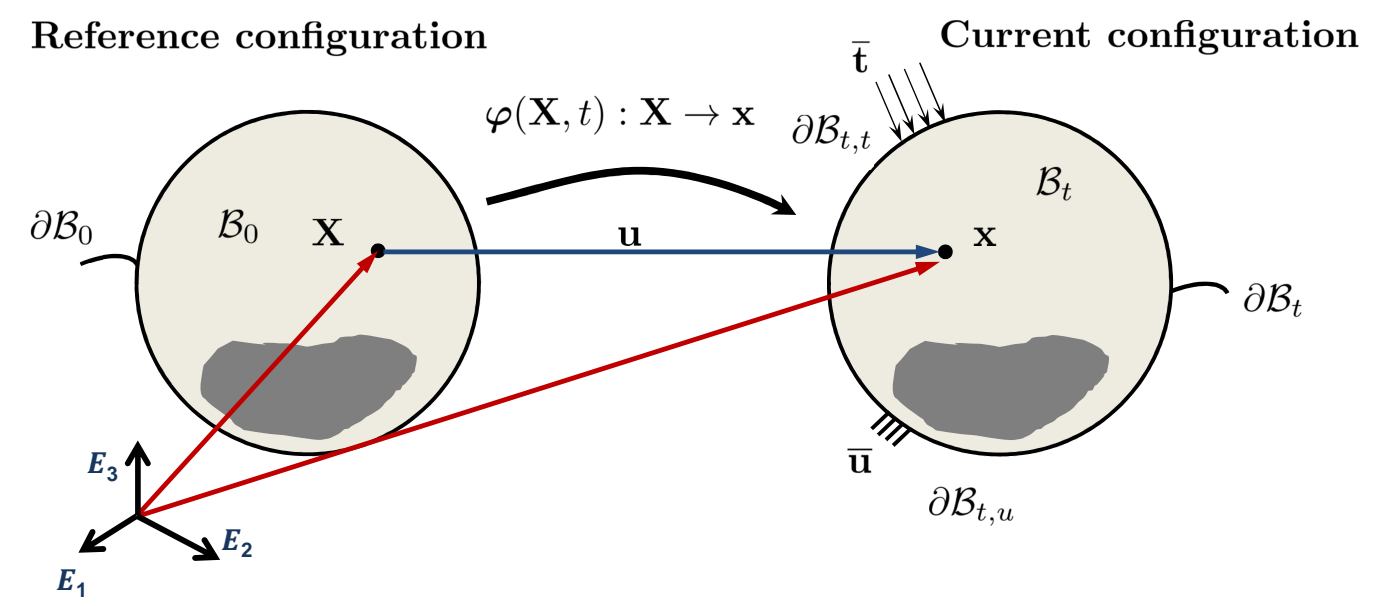

Figure 2: Nonlinear deformation of the solid. Definition of reference $\mathcal{B}_{0}$ and current $\mathcal{B}_{t}$ configurations of the solid. The nonlinear deformation map is defined as $\varphi(\mathbf{X}, t): \mathcal{B}_{0} \times[0, t] \rightarrow \mathbb{R}^{3}$, where $[0, t]$ is the time step interval, and the deformation gradient reads $\mathbf{F}^{u}: \nabla_{\mathbf{X}} \boldsymbol{\varphi}(\mathbf{X}, t)$. The solid is subjected to prescribed static $\overline{\mathbf{t}}$ on $\partial \mathcal{B}_{t, t}$ and kinematic actions $\overline{\mathbf{u}}$ on $\partial \mathcal{B}_{t, u}$.

\subsection{Anisotropic hyperelastic material model}

In the sequel, we address the suitable choices of free energy to set up the constitutive models for anisotropic hyperelastic materials with two families of preferential fibre orientations. In particular, as mentioned above, we consider material models based on Spencer's description [8, whereby the structural tensor is introduced within the framework of invariant-based theory as an additional argument of the Helmholtz free-energy function $\Psi\left(\mathbf{C}, \mathbf{M}_{\mathbf{0}}\right)(i \in 1,2)$. 
As a starting point, the free-energy function can be split into an isotropic and anisotropic counterparts, whereby the embedded fibers are only incorporated into the anisotropic term. The local direction of fiber is described by the unit vector field $\mathbf{a}_{\mathbf{0}} i$ and $\mathbf{a}_{i}$ in the reference and current configurations, respectively. Here $i \in 1,2$ refers to two families of fibers. The material anisotropy is defined by means of the use of the structural tensor, $\mathbf{M}_{\mathbf{0} i}=\mathbf{a}_{\mathbf{0}} \otimes \mathbf{a}_{\mathbf{0}}$, into free energy function. To derive a compact form, it is assumed that the strain energy function of an anisotropic material can be expressed in terms of the invariants right Cauchy-Green tensor $\mathbf{C}$ and the structural tensors $\mathbf{M}_{\mathbf{0} i}$ as follows

$$
\Psi=\Psi\left(\mathrm{I}_{1}, \mathrm{I}_{2}, \mathrm{I}_{3}, \mathrm{I}_{4}^{(i)}, \mathrm{I}_{5}^{(i)}\right) \quad \forall i \in 1,2 .
$$

In expanded form accounting for the previous additive decomposition, Eq. 6. can be expressed as

$$
\Psi\left(\mathrm{I}_{1}, \mathrm{I}_{2}, \mathrm{I}_{3}, \mathrm{I}_{4}^{(i)}, \mathrm{I}_{5}^{(i)}\right)=\underbrace{\Psi^{\text {iso }}\left(\mathrm{I}_{1}, \mathrm{I}_{2}, \mathrm{I}_{3}\right)}_{\text {Rubber matrix }=f\left(\mathrm{I}_{1}, \mathrm{I}_{2}, \mathrm{I}_{3}\right)}+\underbrace{\Psi^{\text {ani }}\left(\mathrm{I}_{4}^{(i)}, \mathrm{I}_{5}^{(i)}\right)}_{\text {Due to the inclusion of } \mathrm{I}_{4}^{(i)} \text { and } \mathrm{I}_{5}^{(i)}} \text {. }
$$

where $\Psi^{\text {iso }}\left(\mathrm{I}_{1}, \mathrm{I}_{2}, \mathrm{I}_{3}\right)$ is the isotropic contribution to the free-energy function, whilst $\Psi^{\text {ani }}\left(\mathrm{I}_{4}^{(i)}, \mathrm{I}_{5}^{(i)}\right)$ identifies the anisotropic term; $\mathrm{I}_{1}, \mathrm{I}_{2}$, and $\mathrm{I}_{3}$ denote the first, second and third invariants of the right Cauchy-Green tensor $\mathbf{C}$, respectively:

$$
\mathrm{I}_{1}:=\operatorname{tr}[\mathbf{C}] ; \quad \mathrm{I}_{2}:=\operatorname{tr}[\operatorname{Cof} \mathbf{C}] ; \quad \mathrm{I}_{3}:=\operatorname{det}[\mathbf{C}],
$$

with $\operatorname{Cof} \mathbf{C}=\operatorname{det}[\mathbf{C}] \mathbf{C}^{-1}$.

In Eq. (7), the fourth invariant is introduced into the free energy function, and its definition reads: $\mathrm{I}_{4}^{(i)}=\operatorname{tr}\left[\mathbf{C M}_{\mathbf{0}_{i}}\right]=\mathbf{C}:\left(\mathbf{a}_{\mathbf{0} i} \otimes \mathbf{a}_{\mathbf{0}}\right)$. The fifth invariant is defined as: $\mathrm{I}_{5}^{(i)}=\operatorname{tr}\left[\mathbf{C}^{2} \mathbf{M}_{\mathbf{0}_{i}}\right]$.

In the case of finite deformation, the fiber stretch $\lambda_{\mathrm{F}_{i}}$ is triggered in terms of deformation gradient $\mathbf{F}$ and the current fiber direction $\mathbf{a}_{i}$ as follows

$$
\lambda_{\mathrm{F}_{i}} \mathbf{a}_{i}=\mathbf{F} \mathbf{a}_{\mathbf{0} i}
$$

Hence with this relation the fiber stretch $\lambda_{\mathrm{F}_{i}}=\sqrt{\mathrm{I}_{4}^{(i)}}$ is the square root of the fourth invariant. Thus at this point, we can introduce the angle between the reinforcing fibres and the matrix $\varphi_{\text {fiber }}^{(i)}$ similar to that defined in Peng et al. [11]:

$$
\cos \varphi_{\text {fiber }}^{(i)}=\sqrt{\frac{1}{\left(\mathbf{a}_{\mathbf{0} i} \cdot \mathbf{C}^{-1} \cdot \mathbf{a}_{\mathbf{0} i}\right)} \frac{1}{\mathrm{I}_{4}^{(i)}}}=\sqrt{\frac{\mathrm{I}_{3}}{\left(\mathrm{I}_{5}^{(i)}-\mathrm{I}_{1} \mathrm{I}_{4}^{(i)}+\mathrm{I}_{2}\right) \mathrm{I}_{4}^{(i)}}} \forall i \in 1,2
$$

where $\mathbf{C}^{-1}=\frac{\mathbf{C}^{2}-\mathrm{I}_{1} \mathbf{C}+\mathrm{I}_{2} \mathbf{1}}{\mathrm{I}_{3}}$.

The introduction of angle $\varphi_{\text {fiber }}^{(i)}$ is important for describing the physical interaction between the fibers and the matrix as will be discussed in Sect. 2.3

Complying with standard arguments within the context of nonlinear Continuum Mechanics, the expression for Cauchy stress $\boldsymbol{\sigma}\left(\mathrm{I}_{1}, \mathrm{I}_{2}, \mathrm{I}_{3}, \mathrm{I}_{4}^{(i)}, \mathrm{I}_{5}^{(i)}\right)(\forall i \in 1,2)$ renders

$$
\begin{aligned}
\boldsymbol{\sigma} & =\mathbf{F}\left(\sum_{k=1}^{3} \frac{\partial \Psi}{\partial \mathrm{I}_{k}} \frac{\partial \mathrm{I}_{k}}{\partial \mathbf{F}}+\sum_{m=4}^{5} \frac{\partial \Psi}{\partial \mathrm{I}_{m}} \frac{\partial \mathrm{I}_{m}}{\partial \mathbf{F}}\right), \\
& =-p \mathbf{1}+2 \Psi_{, \mathrm{I}_{1}} \mathbf{b}+2 \Psi_{, \mathrm{I}_{2}}\left(\mathrm{I}_{1} \mathbf{b}-\mathbf{b}^{2}\right)+2 \Psi_{, \mathrm{I}_{4}} \mathbf{F M}_{\mathbf{0} i} \mathbf{F}^{\mathrm{T}}+2 \Psi_{, \mathrm{I}_{5}} \mathbf{F}\left(\mathbf{C M}_{\mathbf{0}_{i}}+\mathbf{M}_{\mathbf{0}_{i}} \mathbf{C}\right) \mathbf{F}^{\mathrm{T}} .
\end{aligned}
$$

where $\mathbf{b}=\mathbf{F F}^{\mathrm{T}}$ is the left Cauchy-Green tensor and comma denotes to the partial derivative. $p$ is the Lagrange multiplier for incompressibility term when $\operatorname{det}[\mathbf{F}]=1$.

The corresponding elasticity tensor $\mathbb{C}$ is defined as: $\mathbb{C}:=4 \partial_{\mathbf{C}}^{2} \Psi\left(\mathrm{I}_{1}, \mathrm{I}_{2}, \mathrm{I}_{3}, \mathrm{I}_{4}^{(i)}, \mathrm{I}_{5}^{(i)}\right)$. Note that the derivation of the constitutive tensor generally leads to lengthy tensorial computations. 
It is also worth mentioning that following experimental observations, the constitutive formulation is split the deformation locally into dilatational and isochoric counterparts, whereby the deformation gradient can be expressed through the multiplicative composition of isochoric and dilatational factors as follows:

$$
\mathbf{F}=\left[\mathrm{J}^{1 / 3} \mathbf{1}\right] \overline{\mathbf{F}} \text { with } \operatorname{det}[\overline{\mathbf{F}}]=1
$$

Note that from the previous definitions, the computation of the isochoric counterparts of the right and left Cauchy-Green tensors, $\overline{\mathbf{C}}$ and $\overline{\mathbf{b}}$, respectively, can be obtained.

In the sequel, the explicit dependency of the terms of the Helmholtz free-energy functions under consideration is omitted in order to alleviate the notation.

\subsection{Constitutive model of Peng}

The strain energy format proposed by Peng [1] for two fiber families can be divided into three counterparts through the adoption of the following scheme:

$$
\Psi^{\text {Peng }}=\underbrace{\Psi^{\text {iso }}}_{\text {Rubber matrix }}+\frac{1}{2}\left(\Psi^{\text {fiber }}+\Psi^{\mathrm{f}-\mathrm{m}}\right)
$$

where the matrix material is modelled through the use of standard Neo-Hooke potential $\Psi^{\text {iso }}=\Psi^{\mathrm{NH}} ; \Psi^{\mathrm{fiber}}$ identifies the contribution from the fiber stretch and $\Psi^{\mathrm{f}-\mathrm{m}}$ is the strain energy due to the fiber-matrix interaction. The standard form of Neo-Hooke potential is assumed to obey the following expression:

$$
\Psi^{\mathrm{NH}}=\underbrace{\frac{K_{1}}{2}(\operatorname{det} \mathbf{F}-1)^{2}}_{\text {volumetric energy }}+\underbrace{\frac{\mu_{10}}{2}\left(\operatorname{tr}\left[(\operatorname{det} \mathbf{F})^{-2 / 3} \mathbf{F F}^{\mathrm{T}}\right]-3\right)}_{\text {isochoric energy }} .
$$

where $K_{1}$ and $\mu_{10}$ are the bulk and shear modulus, respectively. For the fiber part, a simplified form of free energy potential is assumed as

$$
\Psi^{\text {fiber }}=\mathrm{C}_{2}\left(\mathrm{I}_{4}-1\right)^{2}+\mathrm{C}_{3}\left(\mathrm{I}_{4}-1\right)^{4} \forall \mathrm{I}_{4} \geq 1
$$

Here $\mathrm{C}_{2}$ and $\mathrm{C}_{3}$ are constitutive parameters of the fiber model. The fiber-matrix interaction potential is assumed as

$$
\Psi^{\mathrm{f}-\mathrm{m}}=f\left(\mathrm{I}_{4}\right) \tan ^{2} \varphi_{\text {fiber }}^{(i)} \forall i \in 1,2
$$

The interaction function $f\left(\mathrm{I}_{4}\right)$ is derived based on composite mechanics [1. Without the loss of generality, in this research paper, a sigmoid function is considered in order to account for the interaction function as follows:

$$
f\left(\mathrm{I}_{4}\right)=\frac{\gamma_{\mathrm{s}}}{1+\exp \left[-\beta\left(\lambda_{\mathrm{F}_{i}}-\lambda_{\mathrm{F}_{i}}^{*}\right)\right]} \forall i \in 1,2
$$

where parameter $\gamma_{\mathrm{s}}$ corresponds to the upper limit of $f\left(\mathrm{I}_{4}\right)$. The quantity $\lambda_{\mathrm{F}}^{*}$ is the transition from toe to a linear region in the stress-strain curve, and $\beta$ is a constitutive parameter. The fiber-matrix matrix shear interaction function $f\left(\mathrm{I}_{4}\right)$ for $\gamma_{\mathrm{s}}=12 \mathrm{MPa}$ and $\beta=125$ is depicted in Fig 3 for two different values of $\lambda_{\mathrm{F}}^{*}$.

\subsection{Constitutive model of Balzani}

The second constitutive model herewith analyzed was developed in [9]. According to this modelling framework, the following form of free-energy function is considered for the isotropic part:

$$
\Psi^{\text {iso }}=\underbrace{\epsilon_{1}\left(\mathrm{I}_{3}^{\epsilon_{2}}+\frac{1}{\mathrm{I}_{3}^{\epsilon_{2}}}-2\right)}_{\text {volumetric energy }}+\underbrace{\frac{\mu_{10}}{2}\left(\operatorname{tr}\left[(\operatorname{det} \mathbf{F})^{-2 / 3} \mathbf{F F}^{\mathrm{T}}\right]-3\right)}_{\text {isochoric energy }}, \forall \epsilon_{1}>0, \epsilon_{2}>1
$$




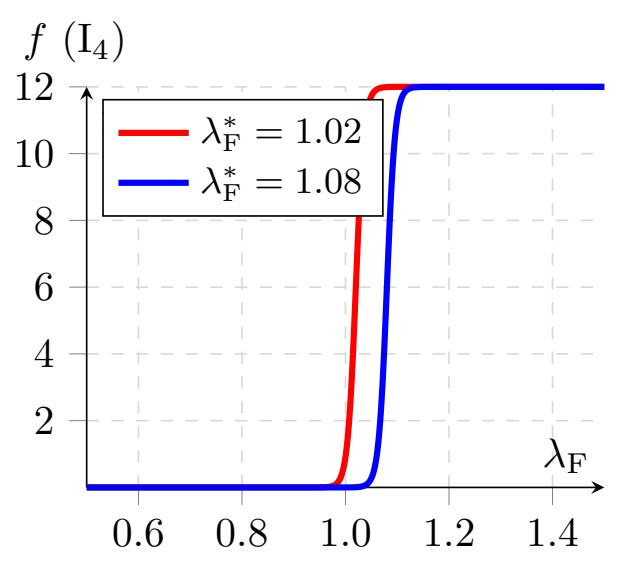

Figure 3: Fiber-matrix interaction factor $f\left(\mathrm{I}_{4}\right)$ as a function of fiber stretch $\lambda_{\mathrm{F}}$ for two different values of $\lambda_{\mathrm{F}}^{*}$.

where $\epsilon_{1}$ and $\epsilon_{2}$ are the constitutive parameters of the model. Particularly, in this investigation, we consider two choices for the polyconvex anisotropic free-energy function, $\Psi_{1}^{\text {ani }}$ and $\Psi_{2}^{\text {ani }}$, which are given by

$$
\Psi_{1}^{\mathrm{ani}}=\sum_{i=1}^{2} \alpha_{3}\left\langle\left(\mathrm{I}_{4}^{(i)}-1\right)^{\alpha_{4}}\right\rangle \forall \mathrm{I}_{4}^{(i)}>1 .
$$

where $\alpha_{1}, \alpha_{2}, \alpha_{3}$ and $\alpha_{4}$ are constitutive parameters.

$$
\Psi_{2}^{\mathrm{ani}}=\sum_{i=1}^{2} \frac{\alpha_{1}}{2}\left\langle\left(\mathrm{~K}_{3}^{(i)}-2\right)^{\alpha_{2}}\right\rangle \forall \mathrm{K}_{3}^{(i)}>2
$$

where $\mathrm{K}_{3}=\mathrm{I}_{1} \mathrm{I}_{4}-\mathrm{I}_{5}$. These two anisotropic terms can be added to the isotropic part, $\Psi^{\text {iso }}$, in order to obtain the total Helmholtz free-energy function of an anisotropic material similarly to that expressed in Eqn.(7). Thus, through the combination of the previous expressions, the following two material modelling choices can be derived:

$$
\begin{gathered}
\Psi^{\mathrm{B}_{1}}=\Psi^{\text {iso }}+\Psi_{1}^{\text {ani }} \\
\Psi^{\mathrm{B}_{2}}=\Psi^{\text {iso }}+\Psi_{1}^{\text {ani }}+\Psi_{2}^{\text {ani }}
\end{gathered}
$$

\section{Virtual Experiment for fiber-reinforced matrix materials}

In this section, with the aim of validating the current FE implementation the anisotropic hyperelastic models outlined in Sect 2.2, we present the simulation of a displacement-driven uniaxial tension test on a specimen manufactured from a fiber-reinforced matrix composite (Fig. 4). In particular, two material orientations, denoted as circumferential specimen and axial specimen are considered with two different relative angles (Fig $4 \mathrm{a}$ ). The external loading applied to the cube and boundary conditions for the virtual tests are depicted in Fig, 4.b, where the preferential material directions are also identified (see the colour version of the plot). The chosen constitutive parameters are reported in Tables 1 and 2 for the Peng [1] (Sect. 2.3) and Balzani [9] (Sect. 2.4) formulations, respectively.

From the numerical point of view, these models have been implemented into the general purpose FE software ABAQUS through the user-defined subroutine UEL. Therefore, a fully implicit numerical solution scheme was employed. To facilitate the programming tasks, the user elements are derived with the AceGen platform [18].

The constitutive response of the Peng model for $2 \varphi_{\text {fiber }}=60^{\circ}$ and $2 \varphi_{\text {fiber }}=120^{\circ}$ are shown in Figs 5 a and 5 .b, respectively. The experimental data for two different fiber angles are shown in Fig 5 by filled circles, 

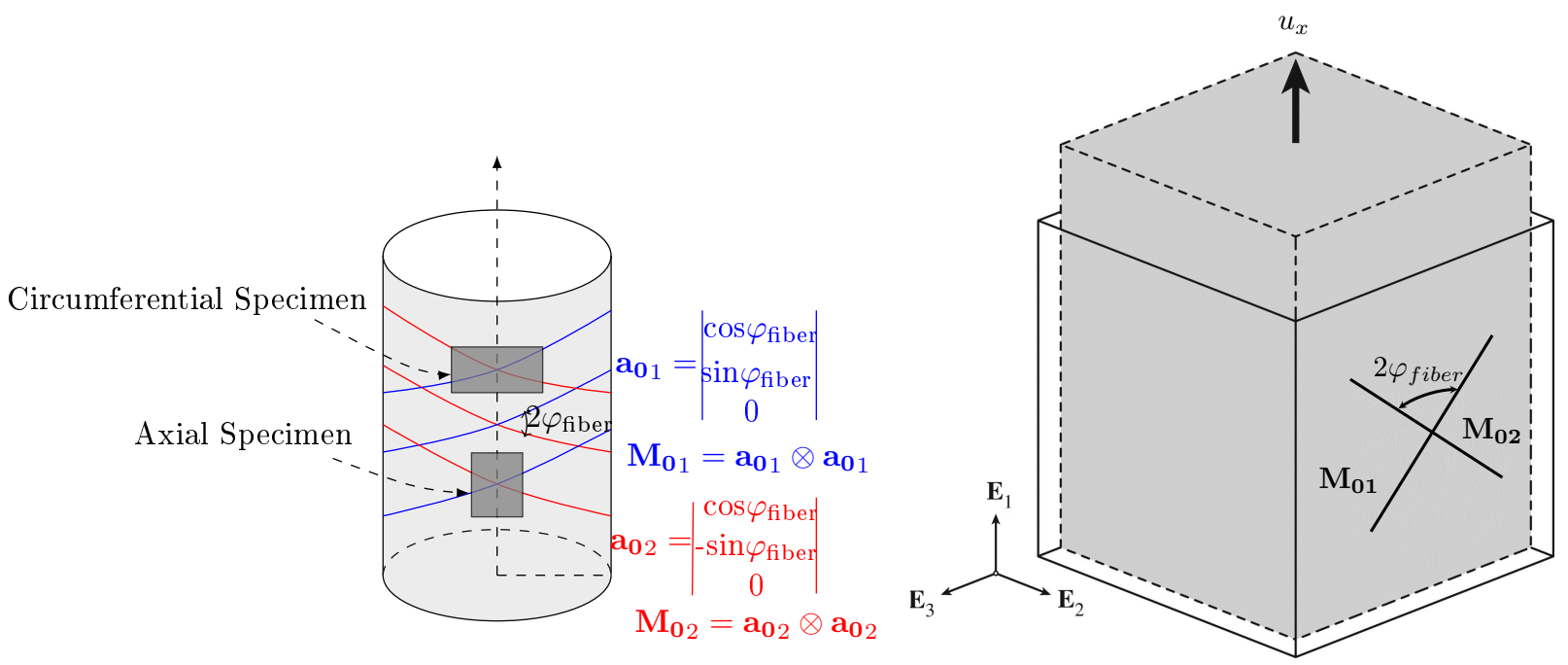

Figure 4: Virtual test under uniaxial tensile loading. (a) Arteries like geometry: anisotropic layer with two embedded families of fibers with mean orientations $\mathbf{M}_{\mathbf{0}_{1}}=\mathbf{a}_{\mathbf{0} 1} \otimes \mathbf{a}_{\mathbf{0}_{1}}$ and $\mathbf{M}_{\mathbf{0}_{2}}=\mathbf{a}_{\mathbf{0} 2} \otimes \mathbf{a}_{\mathbf{0} 2}$ along with the definition of an axial specimen. (b) Uniaxial cube from a longitudinal stripes of a fiber-reinforced matrix, where the angle between fibers is denoted as $2 \varphi_{\text {fiber }}$ that decreases by stretching the material.

Table 1: Constitutive parameters of Peng model

\begin{tabular}{ccc}
\hline Neo-Hooke & Angle & Cord and shear interaction parameter \\
\hline$\mu_{10}=0.068 \mathrm{MPa}$ & $2 \varphi_{\text {fiber }}=60,120 \mathrm{deg}$. & $\mathrm{C}_{2}=0.45 \mathrm{MPa}, \mathrm{C}_{3}=82.6 \mathrm{MPa}$ \\
$K_{1}=10.15 \mathrm{MPa}$ & & $\gamma_{\mathrm{s}}=12 \mathrm{MPa}, \beta=125, \lambda_{\mathrm{F}}^{*}=1.02$ \\
\hline
\end{tabular}

Table 2: Constitutive parameters of Balzani model

\begin{tabular}{ccccccccc}
\hline Free energy & $\mu_{10}$ & $\epsilon_{1}$ & $\epsilon_{2}$ & $\alpha_{1}$ & $\alpha_{2}$ & $\alpha_{3}$ & $\alpha_{4}$ & $\varphi_{\text {fiber }}$ \\
\hline Type & {$[\mathrm{MPa}]$} & {$[\mathrm{MPa}]$} & {$[-]$} & {$[\mathrm{MPa}]$} & {$[-]$} & {$[\mathrm{MPa}]$} & {$[-]$} & {$[$ deg. $]$} \\
\hline$\Psi^{\mathrm{B}_{1}}$ & 35 & 100 & 50 & $5.10^{5}$ & 7 & {$[-]$} & {$[-]$} & 43.39 \\
$\Psi^{\mathrm{B}_{2}}$ & 24 & 100 & 40 & $8.10^{7}$ & 10 & 70 & 2.05 & 43.39
\end{tabular}

whilst the predicted load-displacement curves with and without fiber-matrix interaction factor $f\left(\mathrm{I}_{4}\right)$ are respectively shown with solid and dashed lines. In these graphs it can be observed that the closer agreement between the experimental and the numerical data was obtained setting $f\left(\mathrm{I}_{4}\right)=0$. Nevertheless though an overall satisfactory evolution was predicted, small deviations along the reaction-displacement evolutions can be identified. These discrepancies were ascribed to the definition of a slightly different computational geometry for measuring the response.

The current validation process is extended to the constitutive models described in Sect 2.4. To do so, the polyconvex energies reported by Balzani 9 were fitted against the experimental data presented above for two choices of free-energy functions under consideration in the current research. The corresponding results are depicted in Figs.6a and 6. b. In these graphs, the numerical predictions show an excellent adjustment with respect to the experimental data along the whole reaction-displacement curve, validating the current implementation.

Similar results were obtained using the constitutive model denoted as $\Psi^{\mathrm{B}_{2}}$ in Sect 2.4 see Fig.7 In this evolution, mid deviations of the numerical data with respect to the experimental results can be identified at the end range of the simulations (from $0.22 \mathrm{~mm}$ to $0.27 \mathrm{~mm}$ ) for the case of the longitudinal specimen. At this final stage, the numerical model underestimated the experimental data. 

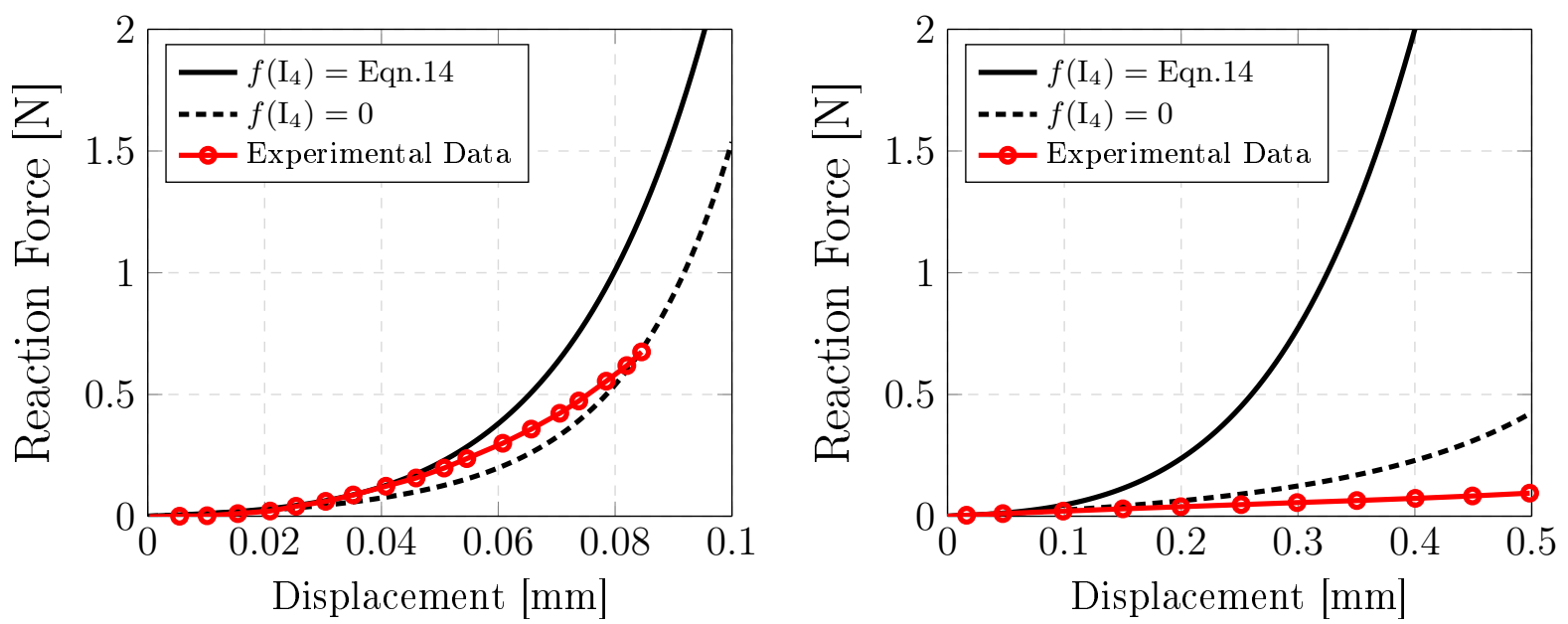

Figure 5: Constitutive response for the Peng model. (a) $2 \varphi_{\text {fiber }}=60^{\circ}$. (b) $2 \varphi_{\text {fiber }}=120^{\circ}$.

Note that the current validation is focused on anisotropic hyperelastic materials for biomechanical applications using a so-called "hand-fitting" process. Nevertheless, these results can be extrapolated to mechanical applications for automotive products in a straightforward manner by simply changing the material parameters. Finally, to conclude this section, it can be observed that the polyconvex energy functions proposed in 9] provided a better adjustment to the experimental data. Therefore, this form for the construction of the free-energy function is explored in the sequel to incorporate the effects of residual stress fields.

\section{Incorporation of residual stresses and damage modelling capabilities}

This Section deals with the incorporation of residual stresses into the previous hyperlastic material models in a consistent and efficient manner. In particular, based on the results reported in Sect 3, our attention is focused on the polyconvex form of the Helmholtz free-energy function proposed in [9] due to their higher accuracy with respect to the experimental results.

\subsection{Constitutive formulation: postulation of the Helmholtz free-energy function}

Residual stresses are often introduced in the engineering structure due to manufacturing processes or in arteries due to nonuniform growth and remodeling processes during development. This is defined as a stress acting on a continuum body which is in equilibrium in the absence of any external loads.

In the related literature, several algorithmic procedures have been proposed in the last years to deal with the incorporation of residual stress fields into material models, cf. [14, 15] and the references therein given. Specifically, in this investigation, a stress-free state is introduced into the current constitutive framework in the sense of Hoger [19] and Merodio and Ogden [16] is adapted.

In line with [16, we assume that the reference configuration is residually stressed and arises in the absence of body forces and surface tractions on the boundary or body actions. The symmetric residual stress tensor is denoted by $\mathbf{T}_{R}\left(=\mathbf{T}_{R}^{\mathrm{T}}\right)$ and satisfies the balance of linear and angular momentum. i.e.

$$
\operatorname{Div} \mathbf{T}_{R}=\mathbf{0} ; \quad \mathbf{T}_{R} \cdot \mathbf{N}=0 \text { on } \partial \mathcal{B}_{0} .
$$

where $\mathbf{N}$ is a normal vector in reference configuration $\mathcal{B}_{0}$. The consideration of this residual stress field affects the overall stress and strain distributions. Moreover, it should be pointed out that residual stresses are necessarily non-uniform and geometry dependent. A residually stressed configuration is shown in Fig. 8. 

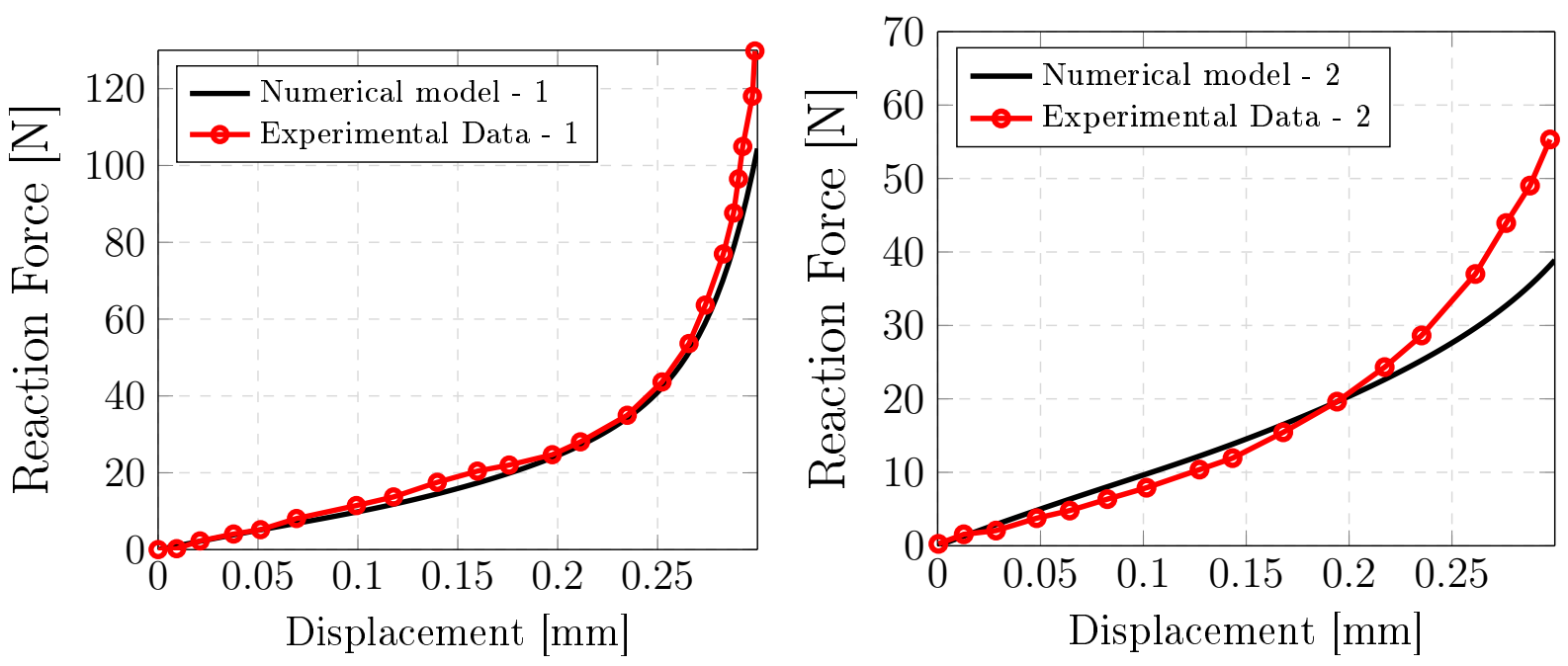

Figure 6: Constitutive response for the model $\Psi^{\mathrm{B}_{1}}$. (a) Characteristic response for the cylindrical specimen. (b) Characteristic response for the longitudinal specimen.

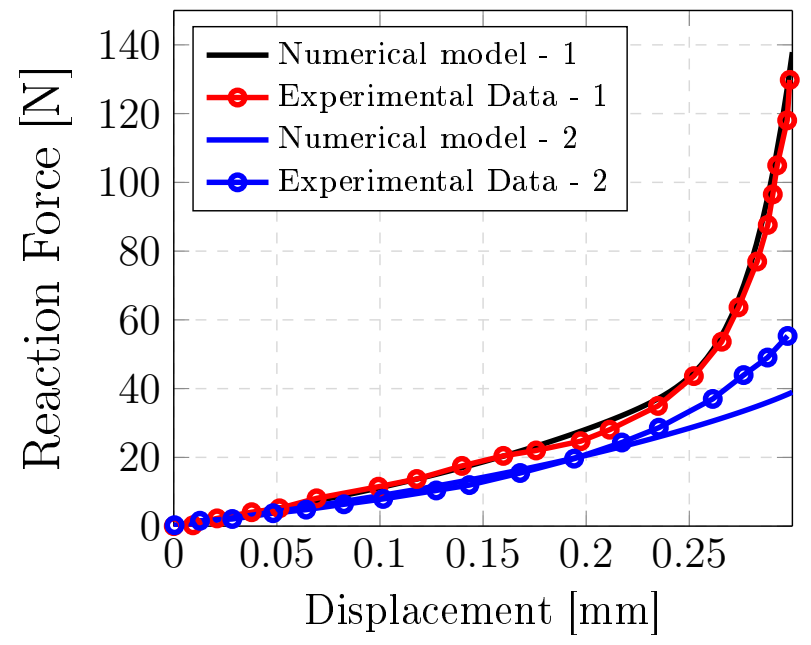

Figure 7: Constitutive response for circumferential and longitudinal specimen at $\varphi_{\text {fiber }}=43.39^{\circ}$ for the material model $\Psi^{\mathrm{B}_{2}}$. 


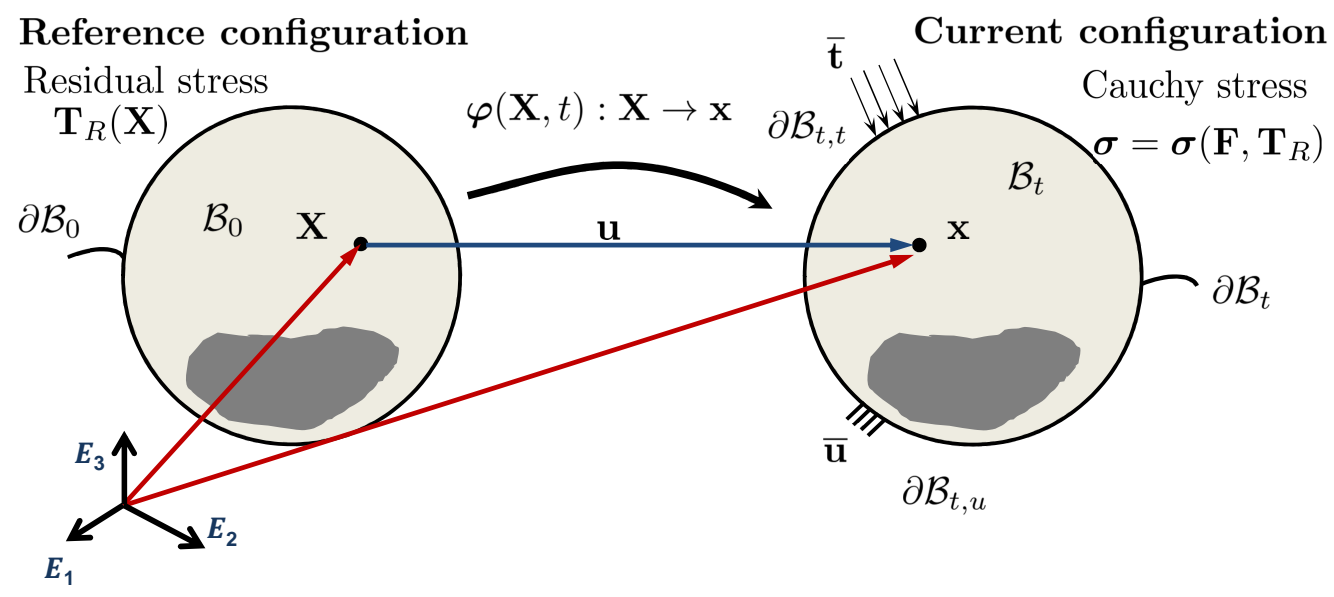

Figure 8: Residually stressed continuum body. Transformation of reference $\mathcal{B}_{0}$ to current Configuration $\mathcal{B}_{t}$.

Thus, under the action of residual stress tensor $\mathbf{T}_{R}$, the expression of free energy takes the following form,

$$
\Psi=\Psi\left(\mathbf{F}, \mathbf{T}_{R}\right)
$$

where $\mathbf{F}$ is deformation gradient. The above expression of free energy is objective since $\mathbf{T}_{R}$ is not affected by rotation in the current configuration $\mathcal{B}_{t}$. Analyzing the structure given in Eq. 24 from a mathematical perspective, it can be observed that the residual stress tensor $\mathbf{T}_{R}$ has a similar role that the structural tensor discussed previously in Sect. 2.4. By performing the spectral decomposition of a given residual stress tensor, one obtains

$$
\mathbf{T}_{R}=\mathrm{T}_{R 1} \mathbf{N}_{R 1} \otimes \mathbf{N}_{R 1}+\mathrm{T}_{R 2} \mathbf{N}_{R 2} \otimes \mathbf{N}_{R 2}+\mathrm{T}_{R 3} \mathbf{N}_{R 3} \otimes \mathbf{N}_{R 3}
$$

where $\mathrm{T}_{R 1}, \mathrm{~T}_{R 2}, \mathrm{~T}_{R 3} \& \mathbf{N}_{R 1}, \mathbf{N}_{R 2}, \mathbf{N}_{R 3}$ are eigenvalues and eigenvectors of residual stress tensor $\mathbf{T}_{R}$, respectively. The eigenvectors are related to each other by the following identity,

$$
\mathbf{N}_{R 1} \otimes \mathbf{N}_{R 1}+\mathbf{N}_{R 2} \otimes \mathbf{N}_{R 2}+\mathbf{N}_{R 3} \otimes \mathbf{N}_{R 3}=\mathbf{1}
$$

Now splitting the free energy expression Eq. 24) into isotropic and residual term as

$$
\Psi=\Psi^{\mathrm{NH}}+\Psi^{\mathrm{rs}}
$$

where $\Psi^{\mathrm{NH}}$ reads similar to Eq. (14) and $\Psi^{\mathrm{rs}}$ identifies the residual contribution to the free-energy. The expression of free energy due to residual related term is given by

$$
\Psi^{r s}\left(\mathbf{T}_{R}\right)=\frac{\alpha_{\mathrm{R}}}{2}\left(\operatorname{tr}\left(\mathbf{T}_{R} \overline{\mathbf{C}}\right)-\operatorname{tr} \mathbf{T}_{R}\right)
$$

where $\alpha_{\mathrm{R}} \in[0,1]$ is a switching parameter related to residual-based free energy.

The substitution of the deformation gradient as an identity tensor into Eqs. (14) and 28), i.e., $\mathbf{F}=\mathbf{1}$, yields a stress-free configuration for a chosen free energy function $\Psi=\mathbf{0}$. Here one can notice that the residual part of free energy $\Psi^{r s}\left(\mathbf{T}_{R}\right)$ resembles with the free-energy expression $\Psi_{2}^{\text {ani }}$ derived eariler in Sec. 2.4 under a suitable choice of parameter.

\subsection{Isotropic damage model}

For the modelling of inelastic process due to damage events, we adopt the isotropic damage approach [20] to the strain energy function, which is characterized by the factor $(1-\zeta)$ with $\zeta$ denoting the damage-like variable. 
In particular, we consider the following modifications in free-energy function, Eq. (27), due to the inclusion of internal variable $\alpha$ (associated with $\zeta$ ) stemming from damage phenomena. The isotropic damage variable affects the isochoric part of elastic strain energy $\Psi^{\mathrm{NH}}$ and the residual parts of the free-energy $\Psi^{r s}\left(\mathbf{T}_{\mathrm{R}}\right)$. The modified free energy reads as :

$$
\Psi\left(\mathbf{F}, \mathbf{T}_{R}, \zeta\right)=(1-\zeta) \underbrace{\left(\frac{\mu_{10}}{2}\left(\operatorname{tr}\left[(\operatorname{det} \mathbf{F})^{-2 / 3} \mathbf{F F}^{\mathrm{T}}\right]-3\right)+\Psi^{r}\left(\mathbf{T}_{R}\right)\right)}_{\Psi_{\text {eff. }}}+\frac{K_{1}}{2}(\operatorname{det} \mathbf{F}-1)^{2}
$$

It is remarked that the adopted damage law is a function of effective energy and mainly acts during tension. Without loss of generality, the following simple expression for the damage evolution law is adopted:

$$
\zeta(\alpha)=1-\mathrm{e}^{-\eta_{d} \alpha} \quad \text { where } \quad \alpha=\Psi_{\text {eff. }}
$$

where $\eta_{d}$ is a constitutive damage parameter. The algorithmic implementation of the constitutive law is very similar to the isotropic damage model proposed in [21], and therefore its detailed algorithmic treatment is omitted here for the sake of brevity. Moreover, the current damage model requires the assumption of a damage criterion, which is defined in the strain space $\Phi_{\mathrm{d}}$ for any arbitrary loading process as

$$
\Phi_{\mathrm{d}}=\mathbb{Y}-\alpha \leq 0
$$

where $\mathbb{Y}=-\frac{\partial \Psi}{\partial \zeta}$ is the thermodynamic force. The evolution of the damage variable $\dot{\alpha}$ is postulated based on maximum dissipation and a constrained optimization problem with the use of Lagrange multiplier $\lambda$ as

$$
\dot{\alpha}=\lambda \frac{\partial \Phi_{\mathrm{d}}}{\partial \mathbb{Y}}
$$

The overall consistent tangent operator in the current configuration $\boldsymbol{c}_{\text {resd }}^{\nabla}$ has been modified due to inclusion of damage law and is expressed for example Case $I$ as

$$
\begin{aligned}
\mathbf{c}_{\mathrm{resd}}^{\nabla} & =(1-\zeta) \mathbb{c}_{\mathrm{iso}}+\boldsymbol{c}_{\mathrm{vol}}-\zeta^{\prime}(\alpha) \mathbb{c}_{\mathrm{dam}} & \text { for } \dot{\mathbb{Y}}>0 \\
& =(1-\zeta) \mathbb{c}_{\mathrm{iso}}+\mathbf{c}_{\mathrm{vol}} & \text { otherwise } .
\end{aligned}
$$

$\zeta^{\prime}(\alpha)=\eta_{d}(1-\zeta)$ is the derivative of damage law with respect to the internal variable $\alpha$ and $\boldsymbol{c}_{\text {dam }}$ is tangent stiffness due to damage variable.

\subsection{Anisotropic damage model}

A very simple extension to the free-energy formulation discussed in Sect. 2.4 is herewith developed in order to incorporate residual stresses into anisotropic solids obeying hyperelastic material laws. In particular, we proposed the following modified Helmholtz free-energy function:

$$
\Psi\left(\mathbf{F}, \mathbf{T}_{R}, \mathbf{M}_{\mathbf{0} i}, \zeta\right)=\Psi^{\mathrm{NH}}+(1-\zeta) \underbrace{\left(\Psi_{1}^{\text {ani }}+\Psi^{r}\left(\mathbf{T}_{R}\right)\right)}_{\Psi_{\text {eff. }}^{\text {aniso }}} .
$$

Thus effective energy release rate for the damage evolution is $\Psi_{\text {eff. }}^{\text {aniso }}=\Psi_{1}^{\text {ani }}+\Psi^{r}\left(\mathbf{T}_{R}\right)$.

Complying with this approach, we derived the corresponding user-defined element of ABAQUS for the given free-energy function. The proposed material model was examined through a simple unit cube subjected to inhomogenous deformation detailed later in sec. 5.1.

\section{Applications: residual stress and damage modeling}

This Section comprises the assessment of the previous constitutive models incorporating residual stress fields presented above. The first case aims at illustrating the effect of residual stresses in standard hyperelastic materials and simple geometries. Subsequently, the proposed formulation is also examined through the use for modeling the response of an isotropic and results regarding its extension to anisotropic materials. In the later part of this section, an opening angle experiments and use of the residual stress model into a cylindrical tube (Sect 5.2) is illustrated. 


\subsection{Application to an isotropic / anisotropic material models}

In this section we study the response of the an applied uniaxial load for two classes of bodies obeying: (i) a isotropic material law, and (ii) an anisotropic material formulation. In both the cases, the continuum body is deforming from an initially residual stressed configuration. For both these cases we examine the cube of unit length. Without the loss of generality we perform the displacement-controlled tension test under the application of displacements in $\mathrm{x}$ direction. The continuum body occupies the region,

$\mathcal{B}=\{(x, y, z) \mid-L \leq x \leq L,-W \leq y \leq W,-H \leq z \leq H\}$, where $L, W$ and $H$ are length, width and height of cube. For illustration, we shall assume that the body is a made of a unit cube, with its dimension $L, W$, and $H=1$ and the initial residual stress in three dimensional Cartesian co-ordinate system in reference configuration is given by,

$\mathbf{T}_{R}=\left(\begin{array}{ccc}\mathrm{T}_{R_{x x}} & \mathrm{~T}_{R_{x y}} & \mathrm{~T}_{R_{x z}} \\ \mathrm{~T}_{R_{x y}} & \mathrm{~T}_{R_{y y}} & \mathrm{~T}_{R_{y z}} \\ \mathrm{~T}_{R_{x z}} & \mathrm{~T}_{R_{y z}} & \mathrm{~T}_{R_{z z}}\end{array}\right)$,

where the initial stress distribution that we develop follows the formulation proposed by PARANJOTHI [? ] setting it extension to $3 \mathrm{D}$ bodies. The corresponding stress field for the cube under consideration is given by

$$
\begin{aligned}
& \mathrm{T}_{R_{x x}}=\mathrm{A}\left[1-\cos \left(\pi \frac{x+L}{L}\right)\right]\left(\cos \left(\pi \frac{y+w}{w}\right)+\cos \left(\pi \frac{z+H}{H}\right)\right), \\
& \mathrm{T}_{R_{y y}}=\mathrm{A}\left[1-\cos \left(\pi \frac{y+W}{W}\right)\right]\left(\cos \left(\pi \frac{X+L}{L}\right)+\cos \left(\pi \frac{z+H}{H}\right)\right), \\
& \mathrm{T}_{R_{z z}}=\mathrm{A}\left[1-\cos \left(\pi \frac{z+H}{H}\right)\right]\left(\cos \left(\pi \frac{x+L}{L}\right)+\cos \left(\pi \frac{y+W}{W}\right)\right), \\
& \mathrm{T}_{R_{x y}}=-\mathrm{A} \sin \left(\pi \frac{x+L}{L}\right) \sin \left(\pi \frac{y+w}{w}\right), \\
& \mathrm{T}_{R_{y z}}=-\mathrm{A} \sin \left(\pi \frac{y+W}{W}\right) \sin \left(\pi \frac{z+H}{H}\right) \\
& \mathrm{T}_{R_{x z}}=-\mathrm{A} \sin \left(\pi \frac{x+L}{L}\right) \sin \left(\pi \frac{z+H}{H}\right) .
\end{aligned}
$$

where $\mathrm{A}$ is the strength of the residual stress with the units of stress per square length. These particular choice of stress components satisfy the equilibrium requirements, see Eq. 23. The analytical surface plot of residual stresses are shown in Fig. 9
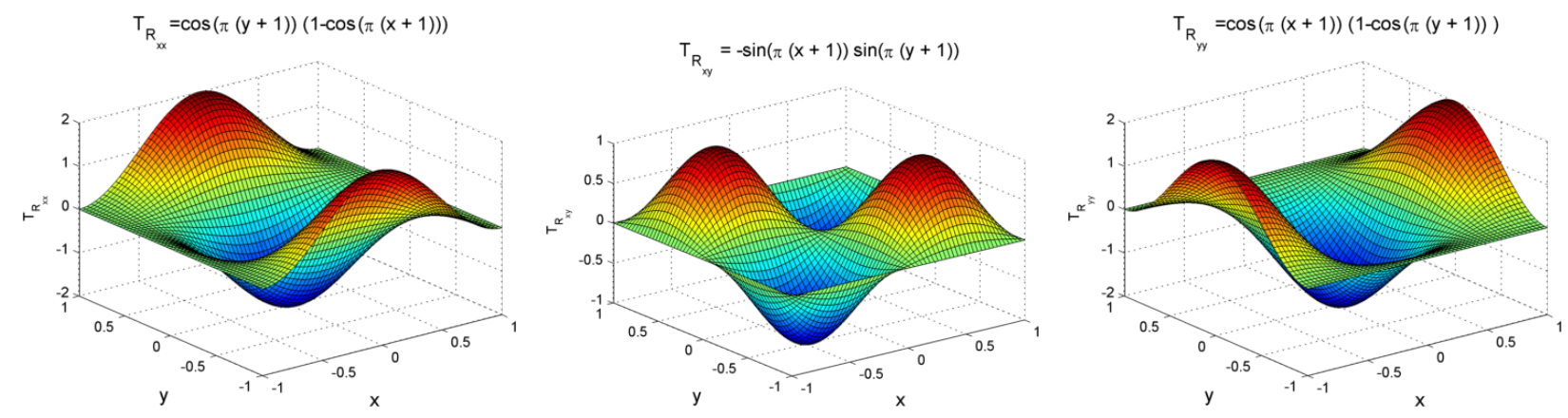

Figure 9: Residual stress over a cube

\subsubsection{Non-homogeneous tension shear test}

Next, the proposed residual stress field is evaluated when solving a three-dimensional (3D) problem. The numerical implementation follows the discussion outlined in Sect. 4.2. Consider non-homogeneous 
displacement loading simultaneously in tension and shear direction as depicted in Fig. 10 . The boundary conditions are such that during the experiment the bottom face of the specimen is fixed where as the top face is subjected to displacement of $u_{y}=1.33 f(t) \mathrm{mm}$ and $u_{z}=0.33 f(t) \mathrm{mm}$, where $f(t)=(1.5 t)^{2}$ is a loading function. The left and right face of the specimen is fixed in x-direction. The constitutive parameters of the rubber block is summarized in Tab. 3. The numerical analyses are performed using the static analysis procedure in ABAQUS/Standard.

Table 3: Constitutive parameters for tension-shear test / Isotropic material

\begin{tabular}{ccc}
\hline Neo-Hooke parameter & Damage & Residual strength parameter \\
\hline$\mu_{10} / 2=1 \mathrm{MPa}$ & $\eta_{d}=\{0,0.02,0.04\} \mathrm{MPa}^{-1}$ & $\mathrm{~A}=0.5 \mathrm{~N} / \mathrm{mm}^{2}$ \\
$K_{1}=105 \mathrm{MPa}$ & & $\alpha_{\mathrm{R}}=1$ \\
\hline
\end{tabular}
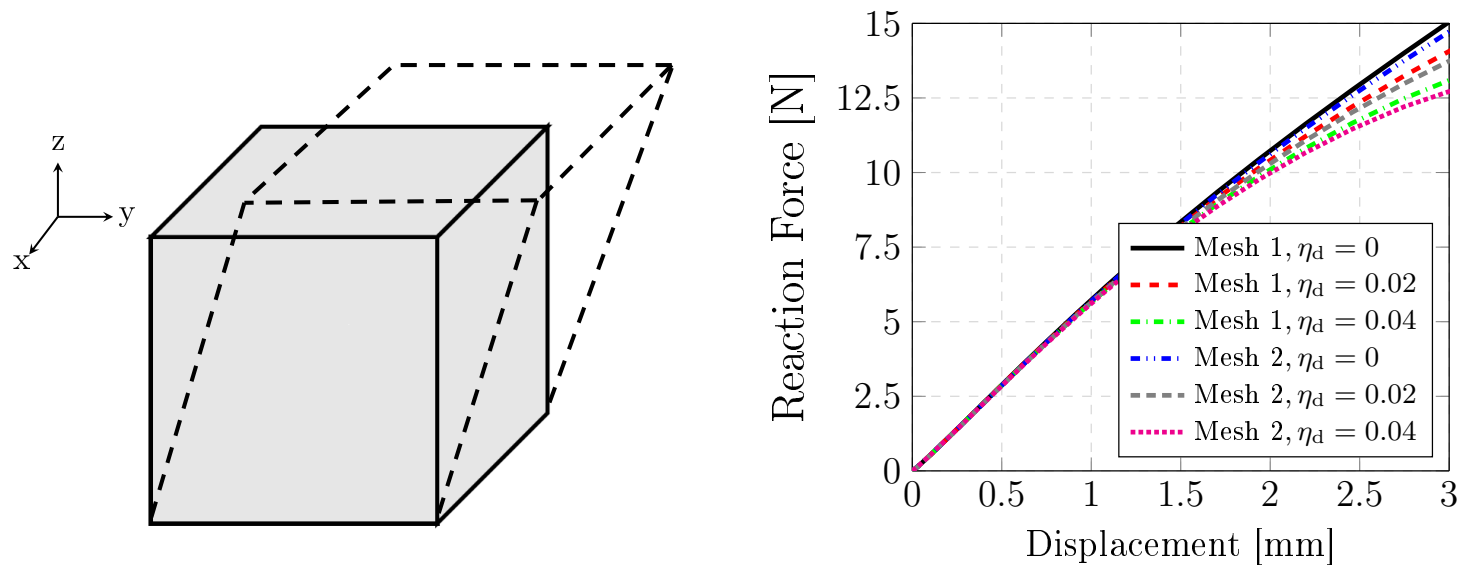

Figure 10: (a) Deformed shapes for one-element tension shear tests (b) Characteristic response for an isotropic material, the resultant reaction force is measured at a point that is constraint through MPC with the top face. The specimen is discretized with Mesh $1=64$ and Mesh $2=1000$ elements.

Figure 10 (b) shows the computed reaction for of a cube for two different mesh sizes and damage parameters $\eta_{d}$. The results are not identical for Mesh 1 and Mesh 2. The stiffer response is observed for coarse mesh. Also, it is noticed that the computed reaction force for higher $\eta_{d}$ is lower. It is primarily due to more degradation at higher damage.

\subsubsection{Extension to anisotropic material models}

Here we study the constitutive response of the developed model detailed in Sect. 4.3. The numerical simulation is carried out under the identical boundary conditions detailed previously in Sec. 5.1.1. The constitutive response for a chosen parameter is portrayed in Fig. 11. Particularly, we discuss the two distinct responses under given constitutive parameters for Mesh 2:

- With stiffening (residual stress) and no damage: The response under this test case was achieved by substituting $\alpha_{\mathrm{R}}=1$ and $\eta_{\mathrm{d}}=0$. The corresponding load capacity refibered at the last converged step was $15.52 \mathrm{~N}$ (black continuous curve in Fig. 11.

- With stiffening (residual stress) and damage: Since damage was activated with stiffening during the loading, the effective energy for the damage evolution in this case was higher for $\eta_{d}=0.04$ than that to $\eta_{d}=0.02$ and corresponding to the previous simulation (green dashed curve in Fig. 11). Hence, the maximum load-capacity was the lowest among the other test cases. 
Table 4: Constitutive parameters of Anisotropic damage model including prestress

\begin{tabular}{ccccccccc}
\hline Free energy & $\mu_{10}$ & $K_{1}$ & $\alpha_{3}$ & $\alpha_{4}$ & $\alpha_{\mathrm{R}}$ & $\mathrm{A}$ & $\eta_{d}$ & $\varphi_{\text {fiber }}$ \\
\hline Type & {$[\mathrm{MPa}]$} & {$[\mathrm{MPa}]$} & {$[\mathrm{MPa}]$} & {$[-]$} & {$[-]$} & {$[\mathrm{MPa}]$} & {$[\mathrm{MPa}]^{-1}$} & {$[\mathrm{deg}]$.} \\
\hline$\Psi\left(\mathbf{F}, \mathbf{T}_{\mathrm{R}}, \mathbf{M}_{\mathbf{0}_{i}}, \zeta\right)$ & 1 & 105 & 10 & 2 & 1 & 0.5 & $\{0,0.02,0.04\}$ & 43.39 \\
\hline
\end{tabular}

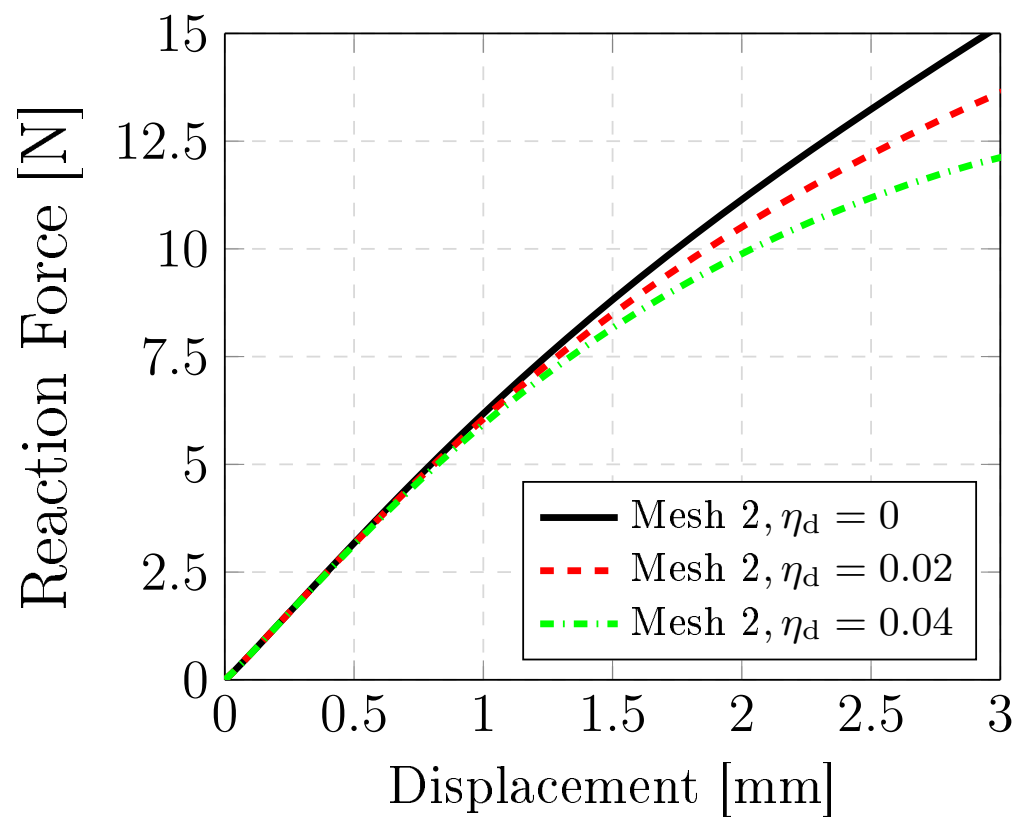

Figure 11: Tensile stress-strain response for the circumferential specimen at $\varphi_{\text {fiber }}=43.39^{\circ}$. for anisotropic damage model

Based on the previous results, the proposed modelling framework led to coherent estimations, allowing the incorporation of residual stress and damage effects into complex material models in a systematic and robust manner.

\subsection{Residual stress in a cylindrical tube}

In this Section, a functional adaptation of a residually stressed tube is demonstrated. Fig. 12 shows the basic geometry under consideration, a tube with outer radius $\mathrm{R}_{o}=2.3 \mathrm{~mm}$, inner radius $\mathrm{R}_{i}=2.1 \mathrm{~mm}$ and length of $0.6 \mathrm{~mm}$ in unloaded configuration that adapted to a new residually stressed configuration under prescribed boundary conditions in radial direction $\sigma_{r r}=-\mathrm{P}$, with $\mathrm{P}$ being the pressure as depicted in Fig. 12. Denoting the residual stresses in radial and azimuthal direction $\mathrm{T}_{\mathrm{RR}}, \mathrm{T}_{\theta \theta}$ respectively, Eqn.23 reduces to

$$
\mathrm{T}_{\mathrm{RR}}=0, \quad \mathrm{~T}_{\theta \theta}=\left(\mathrm{R} \mathrm{T}_{\mathrm{RR}}\right)_{, \mathrm{R}} \text { on } \mathrm{R}=\mathrm{R}_{i}, \mathrm{R}_{o}
$$

where $\mathrm{R}_{i}$ and $\mathrm{R}_{o}$ are the internal and external radii of the tube in the reference configuration $\mathcal{B}_{t}$. A very specific expression of residual stress distribution in a cylindrical co-ordinate system is written as

$$
\mathrm{T}_{\mathrm{RR}}=\mathrm{B}\left(\mathrm{R}-\mathrm{R}_{i}\right)\left(\mathrm{R}_{o}-\mathrm{R}\right) / \mathrm{R}_{i}{ }^{2}, \quad \mathrm{~T}_{\theta \theta}=\mathrm{B}\left(3 \mathrm{R}^{2}-2\left(\mathrm{R}_{o}+\mathrm{R}_{i}\right) \mathrm{R}+\mathrm{R}_{o} \mathrm{R}_{i}\right) / \mathrm{R}_{i}{ }^{2} .
$$

where $\mathrm{R}$ is the material radial coordinate and $\mathrm{B}$ is the strength of a residual field. Distributions corresponding to the stress components $\mathrm{T}_{\mathrm{RR}}$ and $\mathrm{T}_{\theta \theta}$ are depicted in Fig. 12 (right) for $\mathrm{R}_{o}=1.92 \mathrm{~mm}, \mathrm{R}_{i}=1.3 \mathrm{~mm}$, $\mathrm{B}=1 \mathrm{MPa}$ values taken from Ciarletta et. AL. 22.

Finally note that the residual stress distribution considered here led to $\mathrm{T}_{\mathrm{ZZ}}=0$ along the axial direction of the tube. 

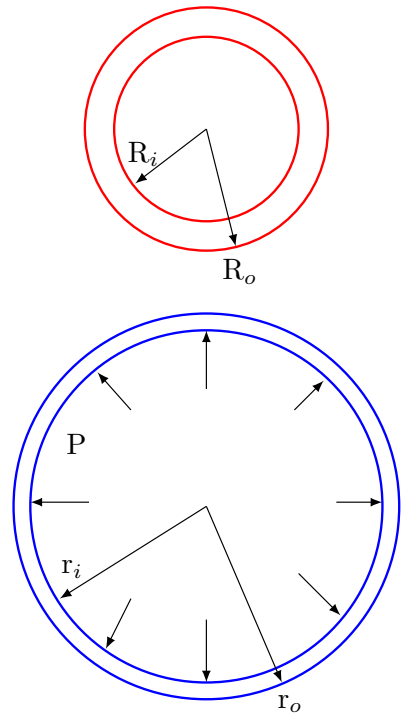

Figure 12: Functional adaptation in tube: change of the unloaded geometry due to internal pressure $\mathrm{P} / \mu_{10}=2.38$ (left); the new adapated geometry is having radius $\mathrm{R}_{i}=1.3 \& \mathrm{R}_{o}=1.92 \mathrm{~mm}$ respectively, and the resulting residual stress distribution as a function of radius $\mathrm{R}$ (right). The radial component $\mathrm{T}_{\mathrm{RR}}=0$ at the inner and outer radii, whereas $\mathrm{T}_{\theta \theta}$ is compressive at inner and tensile at outer surface.

\subsection{Opening angle experiment}

In this section, we address the analysis of an opened-up configuration consisting of a cut section of a ring under the action of residual stresses. A widely used method to obtain the stress-free configuration by so-called "opening angle method" has been demonstrated. Typically the ring opens up releasing residual stresses to the cut-open state as shown in Fig. 13 . The geometric definition of the specimen is: (i) outer radius $\mathrm{R}_{o}=10 \mathrm{~mm},(2)$ inner radius $\mathrm{R}_{i}=8 \mathrm{~mm}$, (iii) thickness $t=2 \mathrm{~mm}$, (iv) initial opening angle $=270^{\circ}$.

In addition to residual stresses, the isotropic damage modeling capability has been also accounted into the constitutive relations as detailed in Sec. 4.2. Preliminary results are carried out in order to minimize the mesh dependence upon the FE discretization, since a local damage formulation is advocated. The final simulations are conducted using 3073 elements C3D8RH of ABAQUS, setting the initial and maximum pseudo-time step equal to 0.02 (Fig,13). Computations are concluded satisfactorily without encountering numerical issues for the achievement of converged states in terms of equilibrium. The residual stress field is released throughout the simulation till reaching a stress-free configuration. The final deformed shape of the system is shown in Fig 14, whereby the final displacement contour is given in Fig. 14.

In line with the previous results, denoting the residual stresses in radial and azimuthal direction $T_{R R}$, $\mathrm{T}_{\theta \theta}$ respectively, the corresponding residual stress field replicates that given in Eq.(36).

In particular, to illustrate the applicability of the proposed formulation, we consider two representative values setting for constitutive damage parameters: The effect of damage parameter on the final state of numerical simulation is depicted in Fig. 15, whereby the final stress-free deformed shape is given. As can be observed in this graph, the current simulations predict that the higher the value for $\eta_{d}$, the higher damage in the specimen is developed. These results are again consistent with the definition of the problem, showing the robustness and applicability of the proposed formulation.

\section{Concluding remarks}

In this paper, a computational modeling framework addressing the incorporation of residual stress and damage effects into fiber-reinforced matrix composite materials has been developed. 


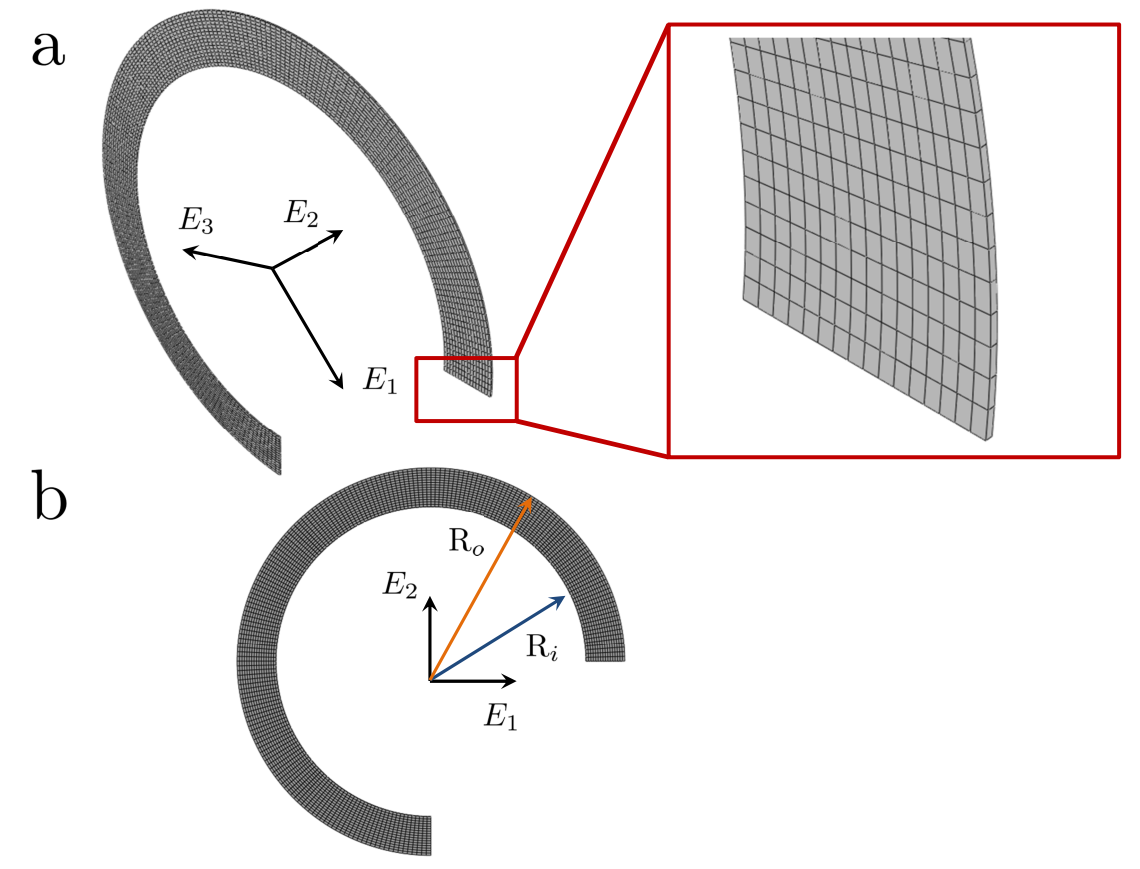

Figure 13: Residually stressed opening angle application. (a) Isometric view and mesh detail. (b) Top view.

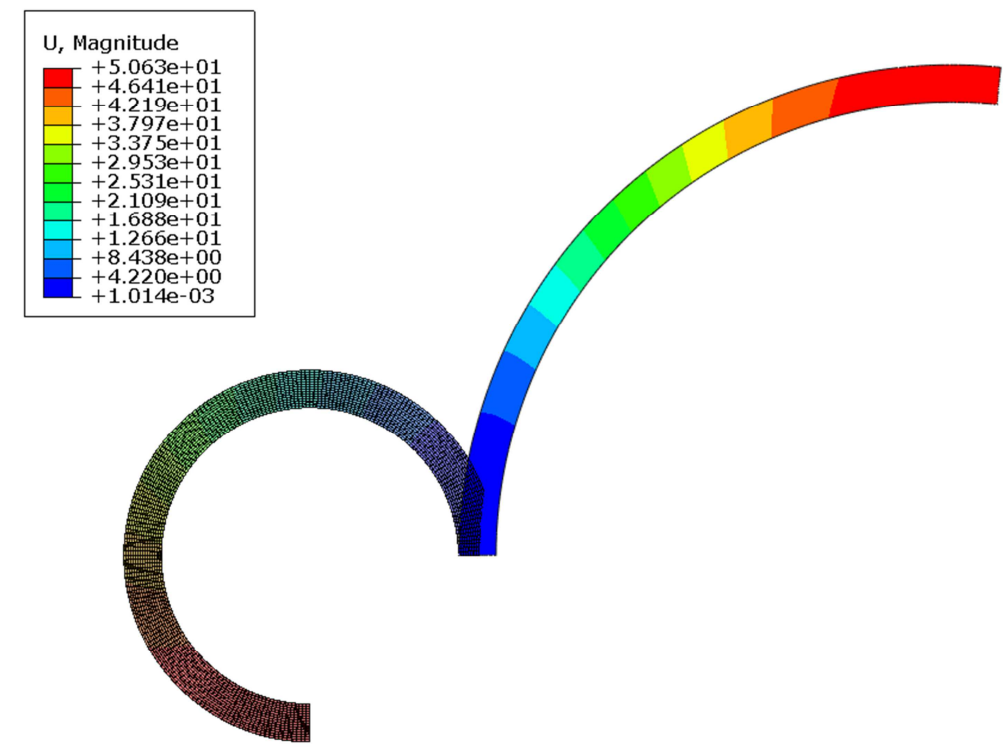

Figure 14: Opening angle: displacement contour and final deformed shape.

One of the main efforts of the current study was the assessment and validation of anisotropic hyperelastic material models with respect to the experimental data. In particular, the current formulation has been derived within the context of the invariant-based tensorial representation [8]. The results herewith discussed 
Figure 15: Damage contour at the last converged step for (a) $\eta_{d}=1$ and (b) $\eta_{d}=25 \mathrm{MPa}^{-1}$ respectively

have evidenced a higher accuracy of the polyconvex material laws proposed in [9] over that developed in [1]

Correspondingly, the current polyconvex material models have been further developed to account for inelastic effects due to damage phenomena. Following the standard isotropic damage formulation in engineering structures affecting the Helmholtz free-energy function by the degradation $(1-\zeta)$ (where $\zeta$ is a scalar-valued damage function), the damage evolution law was formulated in terms of an energy associated with the residual term in the sense of [16, 17.

The second theoretical development has concerned the formulation of a free-energy function which admitted the anisotropic material models together with residual stress and damage effects to be accounted for. This model exploited the additive decomposition of the free-energy function whereby the internal damage parameter was assumed to affect the anisotropic and residual part of the model. This formulation has been verified using a simple uniaxial tensile test.

Finally, relying on the results herewith presented, the current numerical predictions have showed the efficiency and robustness of the proposed computational model, fostering new perspectives for constitutive models which enable the incorporation of inelastic effect from different signature.

Further research activities will comprise the application of the proposed formulation to arteries for studying the instabilities and bifurcation modes, which are of great interest in different engineering applications.

\section{Acknowledgments}

N-K Jha and J Reinoso are grateful to the support of the Spanish Ministry of Economy and Competitiveness/FEDER (Project MAT2015-71036-P).

\section{References}

[1] Xiongqi Peng, Guodong Guo, and Ning Zhao. An anisotropic hyperelastic constitutive model with shear interaction for cordrubber composites. Composites Science and Technology, 78:69 - 74, 2013.

[2] A. Domscheit, H. Rothert, and T. Winkelmann. Refined methods for tire computation. Tire Science and Technology, 17(4):291-304, 1989.

[3] J Reinoso and A Blázquez. A nonlinear finite thickness cohesive interface element for modeling delamination in fibrereinforced composite laminates. Composite Structures, 139:263-276, 2017.

[4] T Brüger, Avon Eitzen, U. Weltin, T. Steinweger, and M. Flamm. Modelling of cord-rubber composites of bellow airsprings. Constitutive Models for Rubber VIII, pages 631-636, 2013.

[5] YH Han, Eric B Becker, Eric P Fahrenthold, and DM Kim. Fatigue life prediction for cord-rubber composite tires using a global-local finite element method. Tire science and Technology, 32(1):23-40, 2004. 
[6] Peter Helnwein, Chang Hong Liu, Günther Meschke, and Herbert A Mang. A new 3-d finite element model for cordreinforced rubber compositesapplication to analysis of automobile tires. Finite elements in analysis and design, 14(1):1-16, 1993.

[7] G Meschke and P Helnwein. Large-strain 3d-analysis of fibre-reinforced composites using rebar elements: hyperelastic formulations for cords. Computational Mechanics, 13(4):241-254, 1994.

[8] A. J. M. Spencer. Theory of invariants. Academic Press, New York, NY, USA, 1971.

[9] Daniel Balzani. Polyconvex anisotropic energies and modeling of damage applied to arterial walls.

[10] G. A. Holzapfel, R. Eberlein, P. Wriggers, and H. W. Weizscker. A new axisymmetrical membrane element for anisotropic, finite strain analysis of arteries. Communications in Numerical Methods in Engineering, 12(8):507-517, 1996.

[11] ZY Guo, XQ Peng, and B Moran. A composites-based hyperelastic constitutive model for soft tissue with application to the human annulus fibrosus. Journal of the Mechanics and Physics of Solids, 54(9):1952-1971, 2006.

[12] A. Dean, S. Sahraee, J. Reinoso, and R. Rolfes. A new invariant-based thermo-plastic model for finite deformation analysis of short fibre reinforced composites: Development and numerical aspects. Composites Part B: Engineering, 125:241 - 258, 2017.

[13] M. Vogler, R. Rolfes, and P.P. Camanho. Modeling the inelastic deformation and fracture of polymer composites part i: Plasticity model. Mechanics of Materials, 59:50 - 64, 2013.

[14] Daniel Balzani, Jörg Schröder, and Dietmar Gross. Simulation of discontinuous damage incorporating residual stresses in circumferentially overstretched atherosclerotic arteries. Acta Biomaterialia, 2(6):609-618, 2006.

[15] D Balzani, J Schröder, and D Gross. Numerical simulation of residual stresses in arterial walls. Computational materials science, 39(1):117-123, 2007.

[16] José Merodio and Ray W Ogden. Extension, inflation and torsion of a residually stressed circular cylindrical tube. Continuum Mechanics and Thermodynamics, 28(1-2):157, 2016.

[17] J. Rodrguez and J. Merodio. Helical buckling and postbuckling of pre-stressed cylindrical tubes under finite torsion. Finite Elements in Analysis and Design, 112:1 - 10, 2016.

[18] J. Korelc. Multi-language and multi-environment generation of nonlinear finite element codes. Engineering with Computers, 18(4):312-327, 2002.

[19] Anne Hoger. On the residual stress possible in an elastic body with material symmetry. Archive for Rational Mechanics and Analysis, 88(3):271-289, 1985.

[20] J. Lemaitre and J. L. Chaboche. Mechanics of solid materials. Cambridge university press, 1994.

[21] G. A. Holzapfel. Nonlinear solid mechanics. Wiley, 2000.

[22] Pasquale Ciarletta, Michel Destrade, and Artur L Gower. On residual stresses and homeostasis: an elastic theory of functional adaptation in living matter. Scientific reports, 6, 2016. 